\title{
COVID-19 Complicated by Concomitant Renal, Splenic, and Myocardial Infarction: Double Whammy
}

\author{
Mehdi Ghaderian Jahromi ${ }^{a} \quad$ Marzieh Sheibani Abdolusefi ${ }^{a}$ \\ Mohsen Neshati Pir Borj ${ }^{b}$ \\ aDepartment of Radiology, Shiraz University of Medical Sciences, Shiraz, Iran; ${ }^{b}$ Department \\ of Cardiology, School of Medicine, Shiraz University of Medical Sciences, Shiraz, Iran
}

\section{Keywords}

Coronavirus disease $2019 \cdot$ Renal infarction · Splenic infarction · Myocardial infarction

\begin{abstract}
A 76-year-old man presented with shortness of breath, fever, and fatigue with confirmed COVID-19 by positive RT-PCR test for the SARS-COV-2. Abdominal and pelvis computed tomography with intravenous contrast was performed several days later given the patient's severe abdominal pain which showed both renal and splenic infarcts despite the use of a proper thromboprophylaxis with subcutaneous heparin, and therefore, therapeutic anticoagulation was initiated. In the course of hospitalization, he developed acute kidney injury, uremic syndrome, and myocardial infarction as well. The patient was treated with fibrinolytic therapy and was eventually discharged on a direct oral anticoagulant.
\end{abstract}

(C) 2021 The Author(s).

Published by S. Karger AG, Basel

\section{Introduction}

The pandemic COVID-19 is caused by severe acute respiratory syndrome coronavirus 2 manifesting as an asymptomatic or mild symptoms to severe pneumonia [1, 2]. Hypercoagulability, in the form of arterial and venous thromboembolism with direct and indirect mechanisms (endothelial injury, stasis, and hypercoagulable state), is a known COVID-19 complication with poor prognostic outcome [1, 3-6].

Herein, we describe a COVID-19 patient with concomitant renal, splenic, and myocardial infarction. To the best of our knowledge, there is no published report consisting concomitant renal, splenic, and myocardial involvement in the context of COVID-19. 


\section{Case Report}

A 76-years-old man, present with 8-day history of shortness of breath, fever, and fatigue. He denied any history of cough, orthopnea, and GI symptoms. In his past medical history, he had asthma and was an ex-smoker. He had no history of underling autoimmune diseases (e.g., antiphospholipid syndrome), acquired hypercoagulability (e.g., cancer), or hereditary thrombophilic disorders. Physical exam and family history were unremarkable. On admission, he was febrile to $38^{\circ} \mathrm{C}$ and tachycardic to 110 beats per minute saturating $75 \%$ on room air, otherwise hemodynamically stable. He had a body mass index of $26 \mathrm{~kg} / \mathrm{m}^{2}$ as well.

Laboratory data showed a WBC of 19,000/UL with lymphopenia, D-Dimer of $0.40 \mathrm{mcg} / \mathrm{mL}$, hCRP $6.0 \mathrm{mg} / \mathrm{dL}$. Renal and liver function tests were within normal range; hepatitis panel was negative.

Considering current pandemic COVID-19, RT-PCR test for the SARS-COV-2 and highresolution computed tomography of lung was performed, which was positive for COVID-19 (shown in Fig. 1). Therefore, a COVID-19 treatment protocol was started including remdesivir, dexamethasone, and subcutaneous unfractionated heparin 5,000 U q12h as a thromboprophylaxis and he transferred to ward.

In the course of hospitalization (8 days later), he developed sudden, constant, and sharp abdominal pain with nausea and vomiting, and then an abdominal and pelvis computed tomography with intravenous contrast was done and revealed, hypodensity of right kidney, and wedge-shape hypodense area in spleen in favor of right kidney and splenic infarction (shown in Fig. 2). On laboratory studies, he had INR 1.0, PT 14.4, partial thromboplastin time 33, D-dimer $1.67 \mathrm{mcg} / \mathrm{mL}$, CRP $8.7 \mathrm{mg} / \mathrm{dL}$, and LDH 4,020 U/L. Hence, he was started on therapeutic anticoagulation with heparin drip.

Two days later, owing to progressive increasing serum creatinine, potassium level, reduced urine output, and significant metabolic acidosis on arterial blood gas, refractory to medical therapy, he underwent emergent hemodialysis and transferred to intensive care unit.

One day later, in spite of improving laboratory studies and general condition, he developed sudden onset severe retrosternal chest discomfort; immediate electrocardiogram was taken which showed anterior ST-segment elevation myocardial infarction. Bedside transthoracic echocardiography was performed and revealed regional wall motion abnormality in anterior wall. Troponin I was also positive. Regarding patient's situation (COVID-19 patient) and also a non-PCI capable hospital, fibrinolytic therapy was administered using tissue plasminogen activator (Actilyse Boehringer ingelheim, Ingelheim am Rhein, Germany) infusion with acceptable result and significant improvement in chest pain. The patient was discharged, 10 days later, on a direct oral anticoagulant and was referred for coronary angiography.

Fig. 1. Multilobar and bilateral ground-glass opacities in both lungs, with a peripheral subpleural distribution.

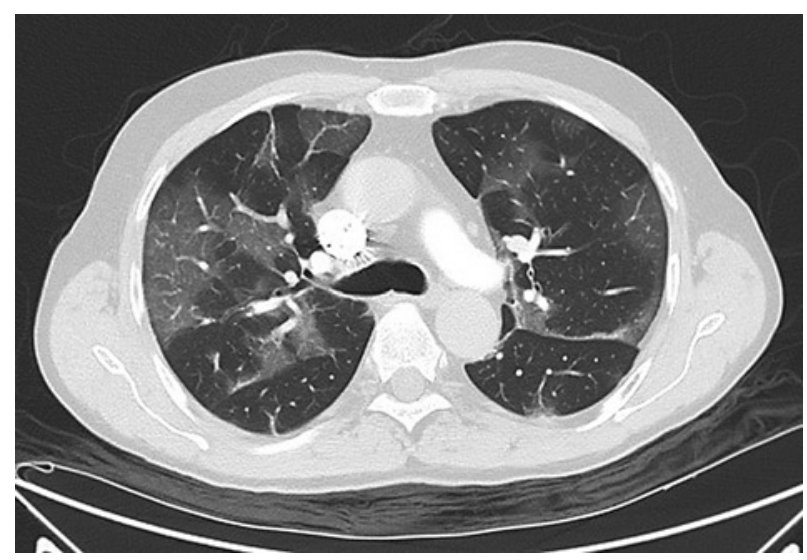


Fig. 2. Renal and splenic infarcts in contrastenhanced CT. Axial (a) and coronal (b) images. Absent enhancement within the majority of the right kidney with a few foci of preserved perfusion (red arrow). Few infarcts appear as hypodense nonenhancing lesions, with normal intervening enhancing splenic tissue (red arrowheads).
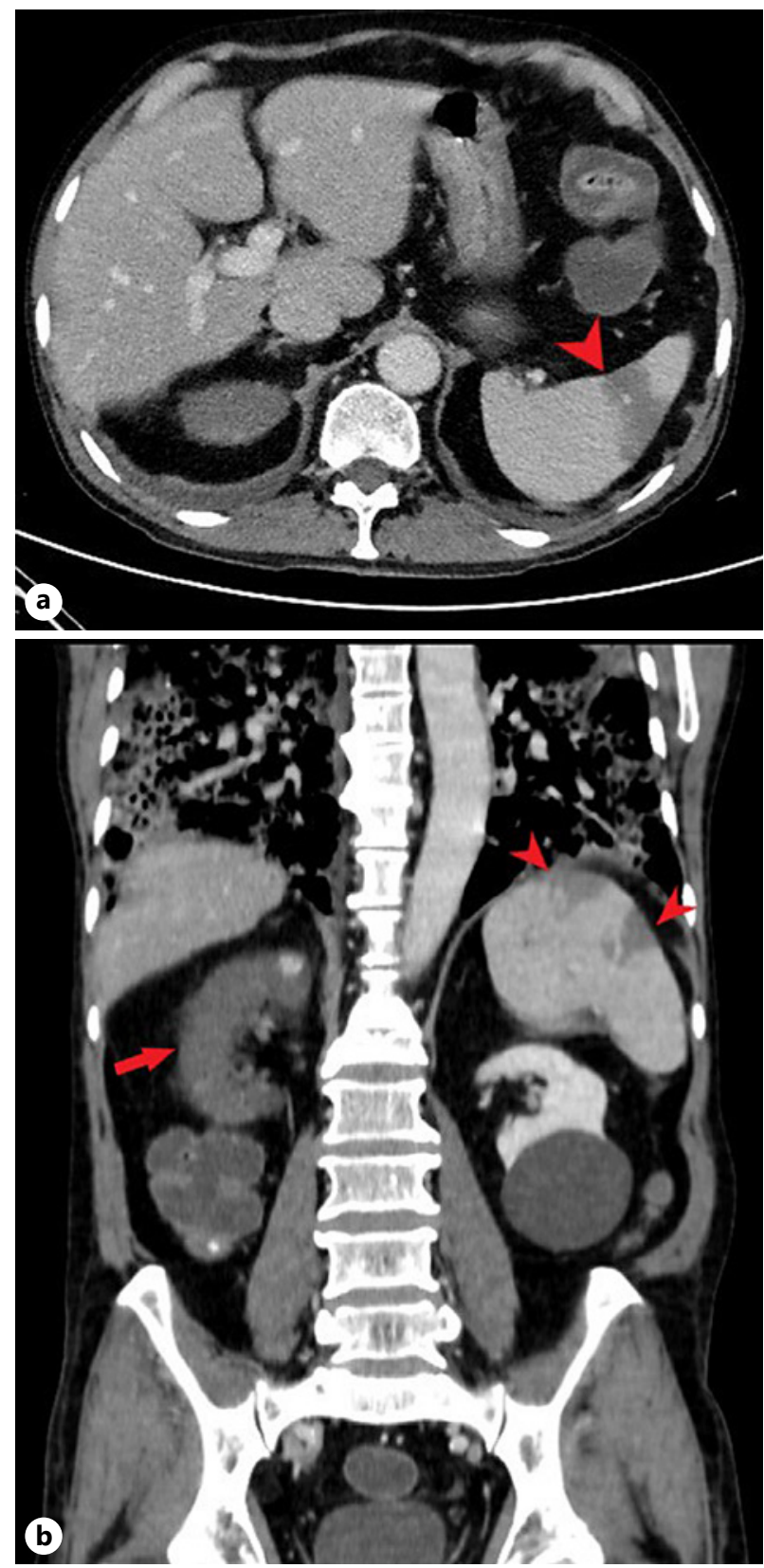

\section{Discussion}

Coagulopathy is a known complication in COVID-19. All 3 components of Virchow's triad seem to be involved, including: endothelitis, stasis, and hypercoagulable state [6]. Venous thromboembolism is common in ill patients with COVID-19 and often occurs as pulmonary embolism and is associated with poor prognostic outcome. High rates of venous thromboembolism events in hospitalized patients with COVID-19 occur despite standard anticoagulation thromboprophylaxis [7-11].

Arterial thrombosis occurs in approximately 4\% of ill COVID-19 patients with multiple arteries involvement in approximately $18 \%$ of patients and is associated with high mortality rate. Arterial thrombotic events are symptomatic in most of patients and lead to limb ischemia, stroke, myocardial infarction, and bowel ischemia [12]. 
Although uncommon, isolated or concomitant renal and splenic infarctions have been reported [13-16]. Guidelines recommend the use of standard prophylactic-dose anticoagulant in all hospitalized COVID-19 patients without a clear contraindication but some note that intermediate-dose anticoagulation can be considered for critically ill patients [17-20]. Nevertheless, Qasim Agha and Berryman [13] reported a case of symptomatic acute splenic thrombosis causing splenic infarction in a patient suffering from a severe case of COVID-19 with morbid obesity despite the use of an intermediate-dose of low-molecular-weight heparin and concluded full-dose or weight-based anticoagulation should be considered in patients with severe COVID-19 and morbid obesity [13].

In present case, not only renal and splenic infarctions occurred despite standard thromboprophylaxis; myocardial infarction developed, while the patient was on therapeutic anticoagulation with unfractionated heparin drip at dose $18 \mathrm{U} / \mathrm{kg} / \mathrm{h}$. To minimize staff risk of COVID-19 exposure, partial thromboplastin time was checked twice a day and all values were within the range of 50-70 s, although it might have been better to keep in a higher range of 60-80 s. What makes our case completely unique and different from previous reports, is myocardial infarction in a COVID-19 patient with multi-organ infarcts (i.e., renal and spleen) under the treatment of therapeutic anticoagulation which necessitates more extended investigation in terms of underling autoimmune diseases, acquired hypercoagulability, and hereditary thrombophilic disorders in such patients irrespective of COVID-19 itself.

In conclusion, although infrequent, arterial thrombosis can occur in patients with COVID-19 in unusual sites including the splenic or renal arteries, and all clinicians should be aware of these complications, and a high degree of suspicion is required in the clinical setting in order to yield the timely diagnosis and treatment. The need of empiric therapeutic-dose anticoagulation or intermediate-dose thromboprophylaxis in COVID-19 patients without a definite diagnosis of thrombosis appears to be still controversial.

\section{Statement of Ethics}

The authors have obtained a written informed consent from the patient to publish his case (including publication of images).

\section{Conflict of Interest Statement}

The author(s) declared no potential conflicts of interest with respect to the research, authorship, and/or publication of this article.

\section{Funding Sources}

This research received no specificgrant from any funding agency in the public, commercial, or not-for-profit sectors.

\section{Author Contributions}

Both M.S. and M.N. wrote the initial draft of the manuscript. M.S., M.N., and M.G. reviewed and participated in the final version of the manuscript. 


\section{References}

1 Zhou F, Yu T, Du R, Fan G, Liu Y, Liu Z, et al. Clinical course and risk factors for mortality of adult inpatients with COVID-19 in Wuhan, China: a retrospective cohort study. Lancet. 2020 Mar 28;395(10229):1054-62.

2 Wu Z, McGoogan JM. Characteristics of and important lessons from the coronavirus disease 2019 (COVID-19) outbreak in China: summary of a report of 72314 cases from the Chinese Center for Disease Control and Prevention. JAMA. 2020 Apr 7;323(13):1239-42.

3 Guan WJ, Ni ZY, Hu Y, Liang WH, Ou CQ, He JX, et al. Clinical characteristics of coronavirus disease 2019 in China. N Engl J Med. 2020 Apr 30;382(18):1708-20.

4 Huang C, Wang Y, Li X, Ren L, Zhao J, Hu Y, et al. Clinical features of patients infected with 2019 novel coronavirus in Wuhan, China. Lancet. 2020 Feb 15;395(10223):497-506.

5 Tang N, Li D, Wang X, Sun Z. Abnormal coagulation parameters are associated with poor prognosis in patients with novel coronavirus pneumonia. J Thromb Haemost. 2020 Apr;18(4):844-7.

6 Singhania N, Bansal S, Nimmatoori DP, Ejaz AA, McCullough AA, Singhania G. Current overview on hypercoagulability in COVID-19. Am J Cardiovasc Drugs. 2020 Oct;20(5):393-403.

7 Spyropoulos AC, Weitz JI. Hospitalized COVID-19 patients and venous thromboembolism: a perfect storm. Circulation. 2020 Jul 14;142(2):129-32.

8 Porfidia A, Valeriani E, Pola R, Porreca E, Rutjes AWS, Nisio MD. Venous thromboembolism in patients with COVID-19: systematic review and meta-analysis. Thromb Res. 2020 Dec;196:67-74.

9 Mondal S, Quintili AL, Karamchandani K, Bose S. Thromboembolic disease in COVID-19 patients: a brief narrative review. J Intensive Care. 2020 Sep 14;8:70.

10 Ren B, Yan F, Deng Z, Zhang S, Xiao L, Wu M, et al. Extremely high incidence of lower extremity deep venous thrombosis in 48 patients with severe COVID-19 in Wuhan. Circulation. 2020 Jul 14;142(2):181-3.

11 Poissy J, Goutay J, Caplan M, Parmentier E, Duburcq T, Lassalle F, et al. Pulmonary embolism in patients with COVID-19: awareness of an increased prevalence. Circulation. 2020 Jul 14;142(2):184-6.

12 Cheruiyot I, Kipkorir V, Ngure B, Misiani M, Munguti J, Ogeng'o J. Arterial thrombosis in coronavirus disease 2019 patients: a rapid systematic review. Ann Vasc Surg. 2021 Jan; 70:273-81.

13 Qasim Agha 0, Berryman R. Acute splenic artery thrombosis and infarction associated with COVID-19 disease. Case Rep Crit Care. 2020 Sep 4;2020:8880143.

14 Santos Leite Pessoa M, Franco Costa Lima C, Farias Pimentel AC, Godeiro Costa JC, Bezerra Holanda JL. Multisystemic infarctions in COVID-19: focus on the spleen. Eur J Case Rep Intern Med. 2020 Jun 3;7(7):001747.

15 Imoto W, Kaga S, Noda T, Oshima K, Mizobata Y, Kakeya H. Coronavirus disease with multiple infarctions. QJM. 2020 Dec;113(12):907-8.

16 Besutti G, Bonacini R, Iotti V, Marini G, Riva N, Dolci G, et al. Abdominal visceral infarction in 3 patients with COVID-19. Emerg Infect Dis. 2020 Aug;26(8):1926-8.

17 Barnes GD, Burnett A, Allen A, Blumenstein M, Clark NP, Cuker A. Thromboembolism and anticoagulant therapy during the COVID-19 pandemic: interim clinical guidance from the anticoagulation forum. J Thromb Thrombolysis. 2020 Jul;50(1):72-81.

18 American Society of Hematology. COVID-19 resources. 2020. Accessed 2020 Aug 3. Available from: https:// www.hematology.org/covid-19.

19 Royal College of Physicians. Clinical guide for the prevention, detection and management of thromboembolic disease in patients with COVID-19. 2020. Accessed 2020 Nov 13. Available from: https://icmanaesthesiacovid-19.org/clinical-guide-prevention-detection-and-management-of-vte-in-patients-with-covid-19.

20 Spyropoulos AC, Levy JH, Ageno W, Connors JM, Hunt BJ, Iba T. Scientific and standardization committee communication: clinical guidance on the diagnosis, prevention, and treatment of venous thromboembolism in hospitalized patients with COVID-19. J Thromb Haemost. 2020 Aug;18(8):1859-65. 